\title{
In Vitro Susceptibility of Gram-Positive Cocci Isolated from Skin and Respiratory Tract to Azithromycin and Twelve Other Antimicrobial Agents
}

Caio M. F. Mendes, Sumiko I. Sinto,

\author{
Fleury Laboratory, São Paulo, SP, Brazil
}

Carmen Paz Oplustil and ResistNet Brazil Group

\begin{abstract}
This study was conducted to evaluate the activity of azithromycin in comparison to 12 other antibacterial agents against recent isolates obtained consecutively from patients with respiratory tract or skin infections, from January to July, 2000. A total of 717 Gram-positive cocci were analyzed in this study and the following species were studied: Staphylococcus aureus $(\mathrm{n}=576), \beta-$ hemolytic streptococci ( $n=115)$, and Streptococcus pneumoniae $(n=26)$. Susceptibility testing was carried out by the disk diffusion method and interpreted according to NCCLS breakpoints. The activity of azithromycin was compared to erythromycin, clindamycin, chloramphenicol, ciprofloxacin, ofloxacin, oxacillin, penicillin, ceftriaxone, tetracycline, trimethoprim/ sulfamethoxazole, teicoplanin, and vancomycin. Of the $26 \mathrm{~S}$. pneumoniae isolates recovered from the respiratory tract, $5(19.2 \%)$ were intermediate resistant to penicillin. All of these strains were susceptible to chloramphenicol, ofloxacin, and vancomycin, and $24(92 \%)$ were also susceptible to azithromycin, clindamycin, and erythromycin. Among the $67 \beta$-hemolytic streptococci strains isolated from the respiratory tract, $66(99 \%)$ were susceptible to azithromycin, erythromycin, clindamycin, and ofloxacin. All $48 \beta$-hemolytic streptococci strains isolated from skin were susceptible to azithromycin and clindamycin, $47(98 \%)$ were susceptible to erythromycin, and $46(96 \%)$ were susceptible to ofloxacin. Of the 576 strains of $S$. aureus, 253 $\mathbf{( 4 3 . 9 \% )}$ were isolated from the respiratory tract and $323(56.1 \%)$ from skin. Among $S$. aureus isolates from the respiratory tract and skin, $46(18 \%)$ and $78(24 \%)$, respectively were resistant to oxacillin. Isolates from the respiratory tract and skin showed the same percentage of resistance $(36 \%)$ to azithromycin. These in vitro results suggest that azithromycin can be a therapeutic option for treatment of infections caused by these bacteria since the newer macrolides have several distinct advantages over erytromycin including improved oral bioavailability, longer halflife allowing once or twice daily administration, higher tissue concentrations and less gastrointestinal adverse effects.
\end{abstract}

Key Words: Gram-positive cocci, azithromycin, respiratory tract infection, skin infection, macrolide.

Newer macrolide antimicrobials are synthesized by alteration of the erythromycin base. They are compounds with improved pharmacokinetics [1], and fewer gastrointestinal side effects [2]. In 1991 and

Received on 3 April 2001; revised 26 June 2001.

Address for correspondence: Dr. Caio M F Mendes. Av. General Waldomiro de Lima, 508, Zip Code: 04344-070, São Paulo-SP, Brazil. E-mail: caio.mendes@fleury.com.br

The Brazilian Journal of Infectious Diseases 2001;5(5):269-276 (C) 2001 by The Brazilian Journal of Infectious Diseases and Contexto Publishing. All rights reserved. 1413-8670
1992, the Food and Drug Administraton (FDA) approved two of these agents, clarithromycin and azithromycin, for clinical use.

Azithromycin is the first of the azalide antibiotics, chemically related to erythromycin but modified by the insertion of a methyl-substituted nitrogen at position 9a of the lactone ring [3]. This structural alteration has resulted in an important change in the pharmacokinetic profile and an excellent antibacterial spectrum, with activity similar to erythromycin against Gram-positive respiratory pathogens $(S$. aureus, $S$. pneumoniae and S. pyogenes) [4]. This structural change also makes 
the compound more stable in acid, significantly increases the serum half-life and tissue penetration, and results in increased activity against many Gram-negative organisms.

Although generally bacteriostatic, azithromycin is bactericidal against $S$. pyogenes, $S$. pneumoniae and Haemophilus influenzae. Resistance appears to correlate with the amount of macrolide use within a community, as evidenced by a decrease in erythromycin resistance among group A streptococci associated with a nationwide decrease in macrolide use [5]. Azithromycin was developed for oral treatment of bacterial infections of the upper and lower respiratory tract caused by organisms such as $S$. pneumoniae and S. pyogenes, skin and skin structure infections caused by $S$. aureus, and S. pyogenes [6].

Erythromycin has been considered to be the main agent for treating Gram-positive streptococcal infections in penicillin-allergic patients. However, increased resistance to erythromycin in S. pyogenes isolates has been reported in several parts of the world $[7,8]$.

Since the development of penicillin resistance in $S$. pneumoniae in the 1960s, the prevalence of resistance to other beta-lactams and other classes of antimicrobial agents has increased worldwide, resulting in clinical problems in the treatment of infections caused by this microorganism, and highlighting the need for alternative therapeutic procedures [9].

Data from the Sentry Antimicrobial Surveillance Program showed that $S$. aureus was the most common etiologic agent causing skin and soft tissue infections in Latin American hospitals, with approximately, 31.6\% and 30.5\% (1997 and 1998, respectively) of the tested isolates showing resistance to oxacillin, and $39.7 \%$ and $46.6 \%$ showing resistance to erythromycin [10].

For effective clinical use of antibiotics, it is important to know epidemiologic data of local and regional bacterial resistance rates. The purpose of this study was to evaluate antimicrobial susceptibility of Gram-positive isolates from respiratory tract and skin to azithromycin and 12 other agents, as part of the ResistNet Brazil Antimicrobial Surveillance Program.

\section{Materials and Methods}

Five centers from the city of São Paulo (Laboratório Fleury, Laboratório de Investigação Médica - LIM 54 HC-FMUSP, Laboratório Especial de Microbiologia Clínica-LEMC-UNIFESP, Hospital Universitário USP and Instituto de Infectologia Emílio Ribas), and 6 other centers around Brazil: Florianópolis (Laboratório Médico Santa Luzia), Salvador (Hospital Aliança), Fortaleza (Laboratório Louis Pasteur), João Pessoa (Laboratório Nucilab), Goiânia (Hospital do Câncer), and Curitiba (Hospital de Clínicas and Hospital Evangélico), were included in this project.

\section{$\underline{\text { Bacterial strains }}$}

A total of 717 Gram-positive cocci isolated from respiratory tract and skin, consecutively collected from January to July, 2000, were analyzed. All isolates were identified at the participating center by the routine methods in use at each laboratory, according to the American Society for Microbiology (ASM) procedures.

\section{$\underline{\text { Susceptibility testing methods }}$}

Antimicrobial susceptibility testing was performed by disk diffusion method, and results were interpreted using the National Committee for Clinical Laboratory Standards (NCCLS ) [11,12]. The testing procedures were standardized by providing common lots of disks (OXOID, Hampshire, England) and quality control strains obtained from the same manufacturer for all centers. External quality control were sent by the coordinating center to all participating centers every 2 months for identification of the microorganism and susceptibility tests.

Prior to initiating the study, each participating laboratory tested quality control organisms, and, if the results were satisfactory for all antimicrobial agents, each center could initiate the study. On each day, quality control testing was performed by each laboratory throughout the study using Staphylococcus aureus ATCC 25923, Streptococcus pneumoniae ATCC 49619 and Enterococcus faecalis ATCC 29212. 
Table 1. In vitro activity of azithromycin and seven other antimicrobial agents against $\beta$-hemolytic streptococci isolated from respiratory tract and skin specimens from 10 centers in Brazil - ResistNet Surveillance Program in Brazil

\begin{tabular}{|c|c|c|c|c|c|c|}
\hline \multirow[b]{2}{*}{ Antimicrobial agents } & \multicolumn{3}{|c|}{ Respiratory Tract $(n=67)$} & \multicolumn{3}{|c|}{ Skin $(n=48)$} \\
\hline & $\% \mathrm{~S}$ & $\%$ I & $\% R$ & $\% \mathrm{~S}$ & $\%$ I & $\% R$ \\
\hline Azithromycin & 99 & - & 1 & 100 & - & - \\
\hline Ceftriaxone & 100 & - & - & 100 & - & - \\
\hline Clindamycin & 99 & - & 1 & 100 & - & - \\
\hline Chloramphenicol & 92 & 4 & 4 & 88 & 10 & 2 \\
\hline Erythromycin & 99 & - & 1 & 98 & 2 & - \\
\hline Ofloxacin & 99 & 1 & - & 96 & 2 & 2 \\
\hline Penicillin & 100 & - & - & 100 & - & - \\
\hline Vancomycin & 100 & - & - & 100 & - & - \\
\hline
\end{tabular}

S: Susceptible; I: Intermediate; R: Resistant.

Table 2. In vitro activity of azithromycin and 8 other antimicrobial agents against $S$. pneumoniae isolated from respiratory tract from 4 centers in Brazil. ResistNet Surveillance Program in Brazil

\begin{tabular}{lrcc}
\hline & \multicolumn{3}{c}{ Respiratory tract (n= 26) } \\
\cline { 2 - 4 } Antimicrobial agents & \% S & \% I & \% R \\
\hline Azithromycin & 92 & - & 8 \\
Clindamycin & 92 & - & 8 \\
Chloramphenicol & 100 & - & - \\
Erythromycin & 92 & - & 8 \\
Ofloxacin & 100 & - & - \\
Penicillin* & 81 & 19 & - \\
Tetracycline & 73 & 12 & 15 \\
Trim/sulfa & 54 & - & 46 \\
Vancomycin & 100 & - & - \\
\hline
\end{tabular}

S: Susceptible; I: Intermediate; R: Resistant; Trim/sulfa: trimethoprim/sulfamethoxazole; *: MIC results. 
Table 3. In vitro activity of azithromycin and 10 other antimicrobial agents against $S$. aureus isolated from respiratory tract and skin from 10 centers in Brazil. ResistNet Surveillance Program in Brazil

\begin{tabular}{|c|c|c|c|c|c|c|}
\hline \multirow[b]{2}{*}{ Antimicrobial agents } & \multicolumn{3}{|c|}{ Respiratory Tract $(n=67)$} & \multicolumn{3}{|c|}{ Skin $(n=48)$} \\
\hline & $\% \mathrm{~S}$ & $\% \mathrm{I}$ & $\% \mathrm{R}$ & $\% \mathrm{~S}$ & $\% \mathrm{I}$ & $\% \mathrm{R}$ \\
\hline Amp/sulbactam & 82 & - & 18 & 76 & - & 24 \\
\hline Azithromycin & 64 & - & 36 & 64 & - & 36 \\
\hline Ciprofloxacin & 82 & 3 & 15 & 79 & 2 & 19 \\
\hline Clindamycin & 74 & 3 & 23 & 74 & - & 26 \\
\hline Erythromycin & 59 & 5 & 36 & 62 & 4 & 34 \\
\hline Oxacillin & 82 & - & 18 & 76 & - & 24 \\
\hline Penicillin & 13 & - & 87 & 9 & - & 91 \\
\hline Teicoplanin & 100 & - & - & 100 & - & - \\
\hline Tetracycline & 75 & 4 & 21 & 64 & 4 & 32 \\
\hline Trim/sulfa & 81 & - & 19 & 73 & - & 27 \\
\hline Vancomycin & 100 & - & - & 100 & - & - \\
\hline
\end{tabular}

S: Susceptible; I: Intermediate; R: Resistant; Trim/sulfa: trimethoprim/sulfamethoxazole.

Table 4. In vitro activity of azithromycin and 9 other antimicrobial agents against $S$. aureus oxacillin susceptible (OXA S) and oxacillin resistant (OXA R), isolated from respiratory tract and skin from 10 centers in Brazil. ResistNet Surveillance Program in Brazil

\begin{tabular}{|c|c|c|c|c|c|c|c|c|c|c|c|c|}
\hline \multirow{3}{*}{$\begin{array}{l}\text { Antimicrobial } \\
\text { agents }\end{array}$} & \multicolumn{6}{|c|}{ Respiratory tract $(n=253)$} & \multicolumn{6}{|c|}{ Skin $(n=323)$} \\
\hline & \multicolumn{3}{|c|}{ OXA S $(n=206)$} & \multicolumn{3}{|c|}{ Oxa $R(n=47)$} & \multicolumn{3}{|c|}{ OXA S $(n=249)$} & \multicolumn{3}{|c|}{ OXA R $(n=74)$} \\
\hline & $\% \mathrm{~S}$ & $\% \mathbf{I}$ & $\% \mathbf{R}$ & $\% \mathrm{~S}$ & $\%$ I & $\% R$ & $\% \mathrm{~S}$ & $\% \mathbf{I}$ & $\% R$ & $\% \mathrm{~S}$ & $\% I$ & $\% R$ \\
\hline Amp/sulbactam & 100 & - & - & - & - & 100 & 100 & - & - & - & - & 100 \\
\hline Azithromycin & 76 & - & 24 & 6 & - & 94 & 81 & - & 19 & 4 & - & 96 \\
\hline Ciprofloxacin & 97 & 1 & 2 & 14 & 4 & 82 & 99 & 1 & - & 9 & 6 & 85 \\
\hline Clindamycin & 89 & 2 & 9 & 13 & - & 87 & 93 & - & 7 & 9 & 1 & 90 \\
\hline Erythromycin & 72 & 4 & 24 & 5 & - & 95 & 78 & 7 & 15 & 4 & - & 96 \\
\hline Penicillin & 15 & - & 85 & - & - & 100 & 12 & - & 88 & - & - & 100 \\
\hline Teicoplanin & 100 & - & - & 100 & - & - & 100 & - & - & 100 & - & - \\
\hline Tetracycline & 90 & 1 & 9 & 17 & 15 & 68 & 80 & - & 20 & 17 & 11 & 72 \\
\hline Trim/sulfa & 95 & - & 5 & 15 & - & 85 & 94 & - & 6 & 1 & 1 & 98 \\
\hline Vancomycin & 100 & - & - & 100 & - & - & 100 & - & - & 100 & - & - \\
\hline
\end{tabular}

S: Susceptible; I: Intermediate; R: Resistant; Trim/sulfa: trimethoprim/sulfamethoxazole. 
$\underline{\text { Antimicrobial agents }}$

S. aureus isolates were tested against azithromycin, erythromycin, clindamycin, ciprofloxacin, oxacillin, penicillin, tetracycline, trimethoprim-sulfamethoxazole, teicoplanin, ampicillin/sulbactam, and vancomycin.

The strains of $\beta$-hemolytic streptococci were tested against azithromycin, erythromycin, clindamycin, ofloxacin, penicillin, ceftriaxone, chloramphenicol, and vancomycin. S. pneumoniae strains were tested against azithromycin, erythromycin, clindamycin, oxacillin (penicillin screening resistance), chloramphenicol, tetracycline, ofloxacin, trimethoprim-sulfamethoxazole, and vancomycin. MIC for penicillin was performed when oxacillin disk diffusion results were below $20 \mathrm{~mm}$. The results from all centers were entered in the Whonet 5 software for further evaluation.

\section{Results}

During the 6 month period, a total of 717 Grampositive isolates were analyzed: $576 \mathrm{~S}$. aureus, $115 \beta$ hemolytic streptococci, and $26 \mathrm{~S}$. pneumoniae were included in the study. Of the total, 59.8\% were isolated from outpatients, and $40.2 \%$ were isolated from inpatients. Of all isolates studied, 371 (51.7\%) were isolated from skin and 346 (48.3\%) were isolated from upper and lower respiratory tract.

Among $\beta$-hemolytic streptococci, 67 (58.3\%) were isolated from respiratory tract, and $48(41.7 \%)$ were isolated from skin. Azithromycin inhibited $100 \%$ of the isolates from skin and $99 \%$ of the strains isolated from respiratory tract. Erythromycin inhibited $99 \%$ and $98 \%$ of the isolates from respiratory tract and skin, respectively. Clindamycin inhibited $99 \%$ and $100 \%$ of the isolates from respiratory tract and skin, respectively. Ofloxacin inhibited $99 \%$ and $96 \%$ of the strains isolated from respiratory tract and skin, respectively. Penicillin, ceftriaxone, and vancomycin inhibited $100 \%$ of all $\beta$ hemolytic streptococci strains (Table 1).

Of the $26 \mathrm{~S}$. pneumoniae strains isolated from respiratory tract, $54 \%$ were isolated from outpatients and $46 \%$ were from inpatients; $81 \%$ susceptible and
$19 \%$ intermediate resistant to penicillin. All strains with intermediate resistance to penicillin were isolated from outpatients, except 1 strain that was isolated from tracheal aspirate of a hospitalized patient. Azithromycin, erythromycin and clindamycin inhibited $92 \%$ of the strains. Tetracycline and trimethoprim-sulfamethoxazole inhibited $73 \%$ and $54 \%$, respectively. All strains were susceptible to ofloxacin, chloramphenicol, and vancomycin (Table 2).

Among $S$. aureus isolates, 323 were isolated from skin, 253 from respiratory tract, $24 \%$ and $18 \%$, respectively, showed resistance to oxacillin. The resistance rate to azithromycin (36\%) was identical for the skin and respiratory isolates. The resistance rates to erythromycin were $36 \%$ and $34 \%$ for isolates from respiratory tract and skin, respectively. The resistance rates for respiratory tract isolates to clindamycin, ciprofloxacin, tetracycline, gentamicin, and trimethoprimsulfametoxazole were lower than those for skin isolates. Vancomycin and teicoplanin inhibited $100 \%$ of all $S$. aureus isolates (Table 3 ).

The 47 of $S$. aureus oxacillin resistant strains and the 206 oxacillin susceptible strains isolated from the respiratory tract, showed a resistance rate to azithromycin of $94 \%$ and $24 \%$, respectively. Considering the $S$. aureus strains isolated from skin, the resistance rate to azithromycin was $96 \%$ for the oxacillin resistant strains $(\mathrm{n}=74)$, and $19 \%(\mathrm{n}=249)$ for the oxacillin susceptible strains (Table 4).

\section{Discussion}

Surveillance studies are extremely important components of any action designed to control the spread of antimicrobial resistance. This study is an important guide to help clinicians choose empirical treatment for these kinds of infections. The Gram-positive cocci are important causes of nosocomial and communityacquired infections, and are sometimes resistant to multiple antimicrobial agents [13]. The number of antimicrobial agents with activity against these microorganisms is limited [14]. Most antimicrobial 
regimens for initial therapy of respiratory and skin infections due to Gram-positive cocci are empiric, and usually consist of orally administered agents.

The increasing resistance of Gram-positive cocci has had an effect on the choice of therapeutic agents for these infections. It is imperative that studies be performed to determine the nature and prevalence of resistance patterns, and these results should be available to clinicians.

There are several recent studies suggesting that the overuse of macrolides might contribute to the high incidence of erythromycin resistance among $S$. pneumoniae and $S$. pyogenes [8]. Resistance to macrolides is observed in many countries, and, in recent studies, the rates of erythromycin resistance in several countries, like Spain, are about $40 \%$ for $S$. pneumoniae and $26 \%$ for $S$. pyogenes [8]. The rates of resistance to azithromycin in Taiwan are about $61 \%$, $100 \%$, and $99 \%$ for penicillin susceptible, intermediate, and resistant pneumococci, respectively [15].

In the present study, among $\beta$-hemolytic streptococci isolated from respiratory tract, $99 \%$ were susceptible to azithromycin and erythromycin, and, among skin isolates, $100 \%$ and $98 \%$ were susceptible to these drugs, respectively. The results of this study are in agreement with previous reports of in vitro susceptibility to macrolides, where the macrolides were significantly more active against $\beta$-hemolytic streptococci strains isolated in Latin America (96\% to $100 \%)$ than those isolated in the United States $(85.8$ to $87.6 \%$ ) or Canada ( $87.7 \%$ to $89.5 \%$ ) [16].

Of the $26 \mathrm{~S}$. pneumoniae isolated, 5 strains (19\%) showed intermediate resistance to penicillin (MIC 0.094 to $1 \mu \mathrm{g} / \mathrm{mL}$ ), and, although the number of strains tested was limited, the incidence of intermediate resistance to penicillin was higher than reported in the LASER study [9] and other studies like Protek, Sentry, and Alexander surveillance studies [17,18]. In this study, resistance to macrolides and clindamycin was found in $8 \%$ of the strains. Among the five strains of $S$. pneumoniae non-susceptible to penicillin, $3(60 \%)$ were susceptible to azithromycin. A high resistance, rate of $46 \%$ to trimethoprim-sulfamethoxazole was observed in both penicillin susceptible and intermediate strains. Other non- $\beta$-lactams agents (chloramphenicol and ofloxacin) showed excellent in vitro activity against all strains.

Among $S$. aureus isolated from skin and soft tissue, $24 \%$ showed resistance to oxacillin, $36 \%$ to azithromycin, $34 \%$ to erythromycin, $26 \%$ to clindamycin, and 19\% to ciprofloxacin. In comparison, based on a 1997 and 1998 study of skin and soft tissue infections in Latin American hospitals, the oxacillin resistant values were $30.5 \%$, erythromycin $39.7 \%$, clindamycin $29.1 \%$, and ciprofloxacin $29.1 \%$ [10]. This slightly lower resistance observed in our study may be due to the fact that most of the $S$. aureus strains were isolated from outpatients (59.8\%). The resistance rates to oxacillin and other antimicrobials for $S$. aureus isolated from respiratory tract were somewhat lower when compared with the strains isolated from skin.

In conclusion, the results of the present study showed low resistance among $S$. pneumoniae and beta-hemolytic streptococci to macrolides even in the face of ever-emerging antimicrobial resistance. Azithromycin also has several distinct advantages over erythromycin including improved oral bioavailability, longer half-life allowing once daily administration, higher tissue concentrations, and less gastrointestinal adverse effects. Analysis of macrolide prescribing and resistance patterns indicates a correlation between increasing macrolide resistance and the increased use of newer, long-acting macrolides, although further study is required to investigate this correlation.

Gram-positive cocci are among the most common agents of several skin and respiratory tract infections. As we observe in this study, there is an important antimicrobial resistance among isolates of Staphylococcus aureus, S. pneumoniae and $\beta$ hemolytic streptococci, as we saw by these results. Furthermore, we demonstrated that important antimicrobial resistance exist among isolates of staphylococci, S. pneumoniae, and $\beta$-hemolytic streptococci. This includes oxacillin resistance among staphylococci, penicillin intermediate resistance, and trimethoprim-sulfamethoxazole resistance among $S$. pneumoniae, and some degree of macrolide resistance among staphylococci. 
Surveillance studies like this one can assist in monitoring bacterial resistance profiles for local analyses as well as multicenter analysis with the use of standardized methods to ensure comparability of results from different centers in the same study and between other surveillance studies.

\section{Acknowledgements}

The authors would like to thank Pfizer Laboratories for supporting the ResistNet Brazil Antimicrobial Surveillance Program. The authors would like to especially thank Dr. Rogério Nunes for his support and review of the manuscript, and to Dr. Heli Ferreira Filho for his cooperation. ResistNet Brazil Group: Caio Mendes, Laboratório Fleury and Laboratório de Investigação Médica - LIM 54 HC-FMUSP, São Paulo; Helio Sader, Laboratório Especial de Microbiologia Clínica (LEMC), UNIFESP, São Paulo; Elsa Masae Mamizuka, Hospital Universitário - USP, São Paulo; Flávia Rossi, Instituto de Infectologia Emílio Ribas, São Paulo; Cássia Zoccoli, Laboratório Médico Santa Luzia, Florianópolis, Santa Catarina; Maria Goreth de Andrade, Hospital Aliança, Salvador, Bahia; Tereza Bandeira, Laboratório Louis Pasteur, Fortaleza, Ceará; Lauro Santos Filho, Laboratório Nucilab, João Pessoa, Paraíba; Vicente Raul Chavarria Irusta, Hospital do Câncer, Goiania, Goiás; Dilair Camargo de Souza e Libera Dalla Costa, Hospital de Clínicas and Hospital Evangélico, Curitiba, Paraná.

\section{References}

1. Pisticelli, S.C., Danziger, L.H., Rodvold, K.A. Clarithromycin and azithromycin: new macrolide antibiotics. Clin Pharm 1992;11:137.

2. Foulds G., Shepard R.M., Johnson R.B. The pharmacokinetics of azithromycin in human serum and tissues. J Antimicrob Chemother 1990;25(suppl A):73.
3. Bright G.M., Nagel A.A., Bordner J., et al. Synthesis in vitro activity of novel 9-deoxo-9 ${ }^{\mathrm{a}}$-aza-9 ${ }^{\mathrm{a}}$ homoerythromycin A derivatives: a new class of macrolides, the azalides. J Antibiot 1988;41:1029-47.

4. Kinasewitz G., Wood R.G. Azithromycin versus Cefaclor in the treatment of acute bacterial pneumonia. Eur $\mathrm{J}$ Clin Microbiol Infec Dis 1991;10:872-7.

5. Seppala H., Klaukka T., Veropio-Varkila J., et al. The effect of changes in the consumption of macrolide antibiotics on erythromycin resistance in group A streptococci in Finland. N Engl J Med 1997;337:441.

6. Hopkins S. Clinical toleration and safety of azithromycin. Am J Med 1991;91(supp. 3A).

7. Seppala H., Nissinen A., Jarvinem H., et al. Resistance to erythromycin in group A streptococci. N Engl J Med 1992;326:292.

8. Betriu C., Redondo M., Palau M. L., et al. Comparative in vitro activities of linezolid, quinupristin-dalfopristin, moxifloxacin, and trovafloxacin against erythromycinsusceptible and resistant streptococci. Antimicro Agents Chemother 2000;44:1838-41.

9. Jacobs M.R., Appelbaum P.C., LASER Study Group. Susceptibility of 1100 Streptococcus pneumoniae strains isolated in 1997 from seven Latin American and Caribbean countries. Int $\mathrm{J}$ Antimicro Agents 2000; 16:17-24.

10. Gales A.C., Jones R. N., Pfaller M. D., et al. Two-year assessment of the pathogen frequency and antimicrobial resistance patterns among organisms isolated from skin and soft tissue infections in Latin American Hospitals: Results from the SENTRY Antimicrobial Surveillance Program, 1997-1998. Int J Infect Dis 2000;4:75-84.

11. National Committee for Clinical Laboratory Standards. Performance standards for antimicrobial disk susceptibility tests. Seventh ed; Approved Standard. (M2-A7) Wayne, PA: National Committee for Clinical Laboratory Standards, 2000.

12. National Committee for Clinical Laboratory Standards. Performance standards for antimicrobial susceptibility testing, Tenth Informational Supplement. (M100-S10). Wayne, PA: National Committee for Clinical Laboratory Standards, 2000.

13. Cormican M.G., Jones R.N. Emerging resistance to antimicrobial agents in Gram positive bacteria, enterococci, staphylococci and non-pneumococal streptococci. Drugs 1996;51(Suppl 1): 6-12.

14. Lewis M.T., Gales A.C., Sader H., et al. Frequency of occurrence and antimicrobial susceptibility patterns for pathogens isolated from Latin American patients with diagnosis of pneumonia: results from the SENTRY antimicrobial surveillance program (1988). Diag Microbiol Infect Dis 2000;37:63-74. 
15. Po-Ren Hsueh, Yung-Ching Liu, Jainn-Ming Shyr, et al. Multicenter surveillance of antimicrobial resistance of Streptococcus pneumoniae, Haemophilus influenzae, and Moraxella catarrhalis in Taiwan during the 19981999 respiratory season. Antimicrob. Agents Chemother 2000;44:1342-5.

16. Pfaller M.A., Jones R.N., Doern G.V., et al. Survey of blood stream infections attributable to Gram-positive cocci: Frequency of occurrence and antimicrobial susceptibility of isolates collected in 1997 in the United States, Canada, and Latin America from the SENTRY antimicrobial surveillance program. Diagn Microbiol Infect Dis 1999;33:283-97.

17. Bartlett J.G., Breiman R.F., Mandell L..A. Communityacquired pneumonia in adults: guidelines for management. Clin Infect Dis 1998;26:811-38.

18. Felmingham D., Gruneberg R.N., the Alexander Project Group. The Alexander Project 1996-1997: latest susceptibility data from this international study of bacterial pathogens from community-acquired lower respiratory tract infections. J Antimicrob Chemother 2000;45:191-203. 